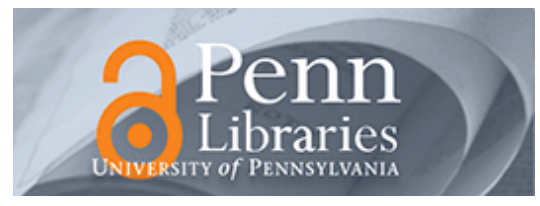

University of Pennsylvania

ScholarlyCommons

Health Care Management Papers

Wharton Faculty Research

\title{
$5-2011$
}

\section{The Medium-Term Impact of Medicare Part D on Pharmaceutical Prices}

Mark Duggan

University of Pennsylvania

Fiona S. Morton

Follow this and additional works at: https://repository.upenn.edu/hcmg_papers

Part of the Health and Medical Administration Commons, and the Insurance Commons

\section{Recommended Citation}

Duggan, M., \& Morton, F. S. (2011). The Medium-Term Impact of Medicare Part D on Pharmaceutical Prices. American Economic Review, 101 (3), 387-392. http://dx.doi.org/10.1257/aer.101.3.387

This paper is posted at ScholarlyCommons. https://repository.upenn.edu/hcmg_papers/77

For more information, please contact repository@pobox.upenn.edu. 


\title{
The Medium-Term Impact of Medicare Part D on Pharmaceutical Prices
}

\begin{abstract}
Medicare Part D began coverage of prescription drugs in 2006. Using data from the first year of the program we found that Part D reduced pharmaceutical prices for Medicare recipients, with these effects driven by enrollees previously without drug coverage. In this paper we extend our analysis through 2009, the fourth year of the program, to investigate whether plans continued to extract price concessions in return for favorable formulary placement, or if consumer inertia or other factors caused prices to bounce back after their initial decline. We find price declines persisted through at least the third year of the program.
\end{abstract}

Disciplines

Health and Medical Administration | Insurance 


\title{
The Medium-Term Impact of Medicare Part D on Pharmaceutical Prices
}

\author{
By Mark G. Duggan and Fiona Scott Morton ${ }^{*}$
}

Part D represents the largest expansion of Medicare since the program's inception in 1965. Though initially projected to cost the federal government $\$ 780$ billion over its first ten years (2006-2015), it has been running substantially under budget. This is due primarily to three factors. The first is that fewer new branded drugs have been invented and launched in the United States, with the result that an increasing fraction of drugs consumed by the elderly are now available in inexpensive generic forms. Second, enrollees have tended to choose less generous plans than was forecasted by government actuaries. Thirdly, Part D is set up so that the government does not directly purchase drugs, but rather subsidizes participating private prescription drug plans (PDPs), which then negotiate with pharmaceutical companies over drug prices. The evidence provided in our previous work suggests that plans have been successful in negotiating lower prices for branded drugs and that this has also helped to contain the cost of the program (Duggan and Scott Morton 2010). In this paper we explore whether these price reductions persisted in the second, third, and fourth years of the program.

The pharmaceutical sales data that we use in this paper indicate that real retail pharmaceutical sales have been growing more slowly in recent years-by just 1.6 percent per year from 2006 to 2009 versus 6.3 percent per year from 2001

\footnotetext{
*Duggan: Department of Economics, University of Maryland, 3105 Tydings Hall, College Park, MD 20742 (e-mail: duggan@econ.bsos.umd.edu); Scott Morton: Yale School of Management, Box 208200, New Haven, CT 06520-8200 (e-mail: fiona.scottmorton@yale.edu). We are grateful to Merck and Co., and especially Anthony Recalde, for providing us IMS data, and to IMS for giving permission for us to use their data. We are also grateful to Wesley Yin for valuable suggestions. This project was supported by National Science Foundation Research Grant 0518858. The opinions contained herein are solely those of the authors. Copyright held by Mark Duggan and Fiona Scott Morton.
}

through 2006. This is a significant change, and it may be that Part D is helping to slow that growth, given Medicare recipients account for more than 40 percent of pharmaceutical spending. However, during this same three-year period, the average monthly premiums paid by Medicare recipients for Part D coverage rose by 8.3 percent annually, suggesting that the price reductions achieved in the first year of the program may not have persisted in subsequent years.

In our prior work, we showed that the institutions and mechanisms used by Part D plans strongly affect market outcomes. By institutions, we have in mind large buyer groups, structured incentives for patients to consume certain products, and the development and use of formularies. A formulary is a mechanism that allows a buyer to identify a therapeutically similar treatment as a viable substitute for a patented treatment, and then create price competition due to the ability to substitute away from the more expensive product. When bargaining with the seller of a patented product, the ability to shift demand to a substitute drug is a powerful negotiating tool.

Our previous research suggests that moving consumers from cash-paying status to membership in a Part D plan lowers optimal prices for branded prescription drugs below what they otherwise would be. This is to some extent surprising because the standard effect of insurance is to create inelastic demand and therefore elicit higher prices from a seller with market power (Duggan and Scott Morton 2006). Furthermore, our estimates reveal the effect is driven by the consumption of drugs by Medicare recipients without insurance before Part D. It appears to be the movement of Medicare recipients from cashpaying uninsured status to insured under a plan

\footnotetext{
${ }^{1}$ Sales, price, and Part D premium data in all years are adjusted to 2009 dollars using the CPI-U. The June 2010 CPI-U is used to adjust dollar values in both 2010 and 2011.
} 
that causes the decline in per unit prices. The most plausible mechanism driving this result is not the insurance per se, but the activities of the insurer. The insurers that we study bundle insurance with a formulary and other mechanisms to create elastic demand.

It is clearly important for policy decisions to determine if the effect on prices was temporary, or if society can continue to expect low prices in Part $\mathrm{D}$ due to the ongoing market pressure that plans place on branded pharmaceutical manufacturers. We explore this issue in the current paper. Our estimation strategy remains the same as in our previous work: we exploit variation across branded drugs in their prepolicy Medicare market shares to estimate the effects of Part D on pharmaceutical prices.

\section{Regulatory Environment and Theory}

Competition in Part D takes place fundamentally at the level of the consumer. A Medicare recipient may choose to enroll in any plan offered in her region of the country. When making this choice, the recipient would consider the plan's monthly premium, the drugs included on the formulary, the prices of those drugs, and any other feature of the plan she values such as brand name or service quality. The plan bargains with drug manufacturers over the prices at which it will purchase drugs on behalf of beneficiaries, who then face out-of-pocket costs for those drugs as described in the plan rules. These price negotiations take place with manufacturers of branded treatments, who have market power due to their intellectual property (patent on the molecule).

To fix ideas, suppose that all Medicare enrollees have no drug coverage prior to Part D and must pay cash for their prescription drugs, but then enroll in Part D when it begins. Notice that when this group paid cash for prescription drugs, its members were not able to create effective price competition between molecules by threatening to switch to a therapeutic substitute. However, once in Part D, these consumers were represented by a PDP. The result of the change in institutional structure is an increase in the cross-price elasticity of substitution for this

\footnotetext{
${ }^{2}$ See Duggan, Patrick Healy, and Scott Morton (2008) for a detailed description of Part D.
}

group. For a number of common models of consumer demand, as the elasticity of substitution rises and other factors remain constant, the optimal price for the product falls. A second effect comes from the fact that the group is now subsidized at 75 percent of the cost of the drug in the main coverage region and 95 percent in the catastrophic region. Sensitivity to price falls and this causes the optimal price to increase. Our earlier results demonstrate that the first effect dominated in 2006, the first year of the program.

While the regulations concerning the operation of Part D have not changed substantially since the start of the program (with respect to drug pricing and negotiation), one can imagine a number of reasons why pharmaceutical prices in Part D may have been unusually low in the first year. It may be that manufacturers initially set low prices to get preferable placement on formularies, but as buyers became less price sensitive they raised prices. The fact that average Medicare Part D premiums ${ }^{3}$ have gone up rapidly suggests that something like this may have occurred. The average annual increase from 2006 to 2010 was 7.5 percent, with an especially large increase of 17.8 percent from 2008 to 2009 (Jack Hoadley et al. 2010). It therefore seems plausible that the price reductions obtained in the first year of the program may not have persisted in subsequent years.

On the other hand, if we think of the buyers of the branded drugs as the Part D plans rather than the ultimate consumers, it is not clear that buyers would become less price-sensitive over time. If consumers have developed switching costs across plans, this would give plans market power, but would not give the plan any reason to bargain less aggressively with the manufacturer. Consistent with this hypothesis is a recent study by the Kaiser Family Foundation that shows considerable variation across (popular) plans in the formulary placement of top brand-name drugs (Elizabeth Hargrave et al. 2010). Many drugs are preferred by a majority of plans, but are not on the formulary at all in other plans. Other drugs are preferred in only a minority of plans and have poor formulary placement in the others. These patterns suggest plans may

\footnotetext{
${ }^{3}$ These premiums cover just 25.5 percent of the cost of standard Part D coverage, with the federal government subsidizing the rest. On the margin, however, consumers face the full cost of choosing more generous plans.
} 
be obtaining price concessions in exchange for formulary placement. As there are theoretical reasons for prices to move in either direction, we provide analysis below to shed light on the question.

\section{Pharmaceutical Sales Data and Constructing the Analysis Sample}

We use data from 2005 to 2009 from IMS Health on total revenues and standardized units of each product (excluding sales to hospitals and long-term care facilities) in the United States for all pharmaceutical products in each year. This allows us to calculate the average price per day in each year for each product. When calculating average prices, we aggregate sales and quantities for all versions of the same drug in the year. Because our data run through 2009, we can estimate the effect of Part D in its first four years.

We merge this IMS data on annual, productspecific average prices to data from the Medical Expenditure Panel Survey (MEPS), which is an annual dataset constructed by the Agency for Healthcare Research and Quality (AHRQ) that includes detailed information on health care utilization for a representative sample of the noninstitutionalized US population. Using the MEPS data, for each drug we calculate the fraction of all prescriptions sold to Medicare recipients in 2002 and 2003, the two years just prior to the passage of the Medicare Modernization Act in December 2003. Approximately 40 percent of all prescriptions are dispensed to Medicare recipients in our two base years but the variation across drugs in this Medicare market share (MMS) is substantial.

Following our previous research on the impact of Medicare Part D (Duggan and Scott Morton 2010), we focus on the top 1,000 drugs in terms of total 2003 revenues. These treatments account for more than 97 percent of all pharmaceutical sales in 2003, with this fraction declining over time, to 87 percent in 2006 and 75 percent by 2009 . This decline occurs because the sample excludes drugs that had not yet been introduced in 2003, and drugs with low initial sales that have high sales later in our study period. We then exclude drugs from the top 1,000 that are generic, sold over the counter, or that do not appear in the 2002 or 2003 MEPS prescribed medicines file. This leaves us with a sample of 545 drugs that have strictly positive sales in 2006. These 545 drugs account for 77 percent of total 2003 pharmaceutical sales, with this share also declining over time to 66 percent in 2006 and 55 percent by 2009 .

One limitation of our focus on these topselling drugs from 2003 is that we will fail to capture any effects of Part D on relatively new products or products with low sales in 2003.

\section{Identification Strategy and Empirical Results}

Merging average prices with information from the MEPS on product-specific Medicare market shares, we estimate specifications of the following type:

$$
\begin{aligned}
\Delta \log \left(P_{j, t}\right)= & \alpha_{1}+\beta_{1} M_{M} S_{j, 2003} \\
& +\mu_{1} \text { Yrs }_{j, 2003} \\
& +\delta_{1} \text { Any }_{\text {Generic }}{ }_{j, 2006} \\
& +\varepsilon_{1 j, t},
\end{aligned}
$$

with $j$ indexing drugs and $\Delta \log \left(P_{j, t}\right)$ equal to the change in the log of the average price for drug $j$ from 2005 to year $t . \square$ In addition to the Medicare market share, this specification controls for the presence of generic competition and for the number of years since the product was approved by the FDA, though our key estimates are not sensitive to the inclusion of these two variables. The coefficient estimate of particular interest is $\beta_{1}$, which captures the average effect of Medicare Part D on pharmaceutical prices. Our key identifying assumption is that there are no unobserved factors correlated with the Medicare market share that also affect pharmaceutical price changes in our time period. By taking first differences of average prices, we remove any unobserved time-invariant differences across drugs. We further assume that any effects of Medicare Part D on average prices are

\footnotetext{
${ }^{4}$ Our earlier paper used one set of data sent to us by IMS for the 2001 through 2006 period. In this paper, we use a second set of data sent to us by IMS for the 2005 through 2009 period. During the three-year period between our receipt of the first and second sets of data, IMS made some changes to the data. Rather than using two different datasets with slight inconsistencies, we use this one dataset that was similarly constructed across all years considered. Thus, our base year is 2005 instead of 2003 as in our earlier paper.
} 
Table 1-The Impact of Medicare Part D on Changes in Pharmaceutical Prices

\begin{tabular}{lcccc}
\hline \hline & $\begin{array}{c}2005-2006 \\
(1)\end{array}$ & $\begin{array}{c}2005-2007 \\
(2)\end{array}$ & $\begin{array}{c}2005-2008 \\
(3)\end{array}$ & $\begin{array}{c}2005-2009 \\
(4)\end{array}$ \\
\hline Medicare market share ${ }_{j, 2003}$ & $\begin{array}{c}-0.104 * * \\
(0.045)\end{array}$ & $\begin{array}{c}-0.258^{* * *} \\
(0.081)\end{array}$ & $\begin{array}{c}-0.180 \\
(0.132)\end{array}$ & $\begin{array}{c}-0.010 \\
(0.163)\end{array}$ \\
Observations & 545 & 545 & 537 & 529 \\
\hline *** Significant at the 1 percent level. & & & \\
** Significant at the 5 percent level. & & & \\
* Significant at the 10 percent level.
\end{tabular}

driven by changes in the prices sold to Medicare recipients rather than prices in other segments of the market. ${ }^{5}$ To the extent that Part D plans succeeded in negotiating lower prices, the estimate for $\beta_{1}$ would be negative.

It is worth emphasizing that our measure of Medicare market share represents the share of prescriptions sold to Medicare recipients before the start of Part D. Not all Medicare recipients are enrolled in Part D, and thus $M M S_{j, 2003}$ may be more appropriately thought of as potential Part D enrollment. Enrollment in Part D increased during our study period, from 22.6 million in August 2006 to 27.0 million three years later, with this latter enrollment representing approximately 60 percent of all Medicare recipients. All else equal, this growth in Part D enrollment would potentially lead to an increasing effect of $M M S_{j, 2003}$ over time.

Columns 1 through 4 of Table 1 display the coefficient on $M M S$ in specifications for the four (average log) price changes from the base year to 2006 to 2009. Consistent with our past work, we find a significantly negative effect of Medicare Part D in the first year of the program, as summarized in column 2. More specifically, the estimate of -0.104 for $\beta_{1}$, which is significant at the 5 percent level, suggests that Medicare recipients experienced price reductions of approximately 10 percent in the first year of the program relative to what they otherwise would have paid. The corresponding coefficient estimate from our earlier paper was almost identical.

In the second column we report the results from specifications with the two-year price change from 2005 to 2007 . The estimate of

\footnotetext{
${ }^{5}$ Recent research by Darius Lakdawalla and Wesley Yin (2010) suggests that Part D increased the negotiating power of health insurers and allowed them to also negotiate lower prices for their non-Medicare recipients.
}

-0.258 is more than twice as large as the oneyear price change and is significant at the 1 percent level. This result provides evidence that there were additional price reductions obtained by Medicare Part D plans in the second year of the program, and that these were similar in magnitude to the declines in year one. In an analogous specification not reported here of the one-year price change from 2006 to 2007, the estimate for $\beta_{1}$ is -0.155 , which is significant at the 1 percent level.

In column 3 we show that the estimate for $\beta_{1}$ in the specification of the three-year price change from 2005 to 2008 remains negative at -0.180 . However, this estimate is not statistically significant. But the somewhat smaller magnitude of this point estimate suggests that there were no further reductions in prices in the third year of the program. In the fourth and final specification, the point estimate for $\beta_{1}$ increases and is close to zero at just -0.010 . However, given a standard error of 0.163 , our estimates are compatible with a wide range of effects by the fourth year of the program. Additionally, the share of all pharmaceutical sales accounted for by our sample is quite low by this final year.

As in our previous paper, we next distinguish between Medicare recipients who already had drug coverage prior to Part D and their counterparts on Medicare who were without this insurance by estimating specifications of the following type:

$$
\begin{aligned}
\Delta \log \left(P_{j, t}\right)= & \alpha+\beta_{1} \text { MMS_Self } \\
& +\beta_{2,2003} \text { MMS_Ins }_{j, 2003} \\
& +\mu \text { Yrs }_{j, 2003} \\
& +\delta_{1} \text { AnyGeneric }_{j, 2006}+\varepsilon_{j, t} .
\end{aligned}
$$


Table 2-Differentiating Between Medicare Recipients WITH AND WITHOUT $\mathrm{R}_{\mathrm{X}}$ INSURANCE

\begin{tabular}{lcccc}
\hline \hline & $2005-2006$ & $2005-2007$ & $2005-2008$ & $2005-2009$ \\
& $(1)$ & $(2)$ & $(3)$ & $(4)$ \\
\hline Medicare self-pay share ${ }_{j, 2003}$ & $-0.144 * * *$ & $-0.278 * * *$ & $-0.372 * *$ & -0.185 \\
& $(0.046)$ & $(0.075)$ & $(0.179)$ & $(0.202)$ \\
Medicare insured share ${ }_{j, 2003}$ & -0.015 & -0.215 & 0.237 & 0.371 \\
& $(0.097)$ & $(0.225)$ & $(0.233)$ & $(0.281)$ \\
Observations & 545 & 545 & 537 & 529 \\
\hline *** Significant at the 1 percent level. \\
** Significant at the 5 percent level. \\
*Significant at the 10 percent level.
\end{tabular}

In this regression, $M M S \_S e l f_{j, 2003}$ is the share of prescriptions dispensed to Medicare recipients who were without drug coverage in 2003, and $M M S_{-}$Ins $_{j, 2003}$ is the share dispensed to beneficiaries with some drug coverage (thus, the two sum to $\left.M M S_{j, 2003}\right)$. Our earlier results demonstrated that the price reductions were driven by this latter group.

Consistent with this, the first column of Table 2 shows that the estimated effect for "uninsured" Medicare recipients in the 2005 to 2006 price change specification is significantly negative, while the corresponding estimate for their insured counterparts is much smaller in magnitude and statistically insignificant. This pattern persists in the subsequent specifications, with the estimate being twice as large through 2007 and even greater through 2008, though the precision of the estimates declines as in Table 1. In all four cases, the estimate for $\beta_{1}$ is negative, and in three cases it is statistically significant. In contrast, the estimate for $\beta_{2}$ is positive in two specifications and negative in two specifications, and is not significant in any of them.

These results suggest that the substantial price reductions for the uninsured that we estimated in the first year of Medicare Part D were even larger in the second and third years of the program. However, the smaller negative coefficient that we estimate in year four suggests simply that the initial price reduction we estimated in 2006 has persisted to 2009.

\footnotetext{
${ }^{6}$ In the specification in which $\beta_{1}$ is not significant, the $p$-value on the difference between $\beta_{1}$ and $\beta_{2}$ is 0.109 .
}

\section{Conclusions}

Our findings in this paper indicate that the Part D plans succeeded in negotiating substantially lower prices for Medicare recipients through the first four years of the program. We estimate additional price declines in years two and three that appear to have been reversed in year four (2009), though we note that the precision of our results declines over time and thus we put less weight on these later estimates.

Our results indicate that the increase in Part D premiums during our study period is not driven by existing brands. One possible explanation is that newly released brands-brands that are not in our sample - are driving up the cost of Part D and leading to higher premiums. A second explanation is that in later years of the program consumers have a cost of switching plans and become less price-sensitive to premiums, and therefore plans have market power. If this explanation were the case, then premiums would be rising due to the market power of Part D plans, not the market power of pharmaceutical manufacturers. A third potential explanation is that Medicare recipients are gravitating toward more generous plans over time. Given the growing importance of Medicare Part $\mathrm{D}$ due to the aging of the baby boom generation and the changes in Part D resulting from the recent health reform legislation, more work in this area is clearly warranted.

\section{REFERENCES}

D Duggan, Mark, Patrick Healy, and Fiona Scott Morton. 2008. "Symposium: Health Care: Providing Prescription Drug Coverage to the Elderly: America's Experiment with Medicare 
Part D." Journal of Economic Perspectives, 22(4): 69-92.

-Duggan, Mark, and Fiona Scott Morton. 2010. "The Effect of Medicare Part D on Pharmaceutical Prices and Utilization." American Economic Review, 100(1): 590-607.

-Duggan, Mark, and Fiona Scott Morton. 2006. "The Distortionary Effects of Government Procurement: Evidence from Medicaid Prescription Drug Purchasing." Quarterly Journal of Economics, 121(1): 1-30.

Hargrave, Elizabeth, Jack Hoadley, Laura Summer, Juliette Cubanski, and Tricia Neuman.
2010. "Medicare Part D Data Spotlight: Coverage of Top Brand-Name and Specialty Drugs." Kaiser Family Foundation. http://kff.org/ medicare/upload/8095.pdf.

Hoadley, Jack, Juliette Cubanski, Elizabeth Hargrave, Laura Summer, and Tricia Neuman. 2010. "Medicare Part D Spotlight: Part D Plan Availability in 2011 and Key Changes Since 2006." Kaiser Family Foundation. http://www. kff.org/medicare/upload/8107.pdf.

Lakdawalla, Darius, and Wesley Yin. 2010. "Insurers' Negotiating Leverage and the External Effects of Medicare Part D." Unpublished. 


\section{This article has been cited by:}

1. Mark Duggan, Craig Garthwaite, Aparajita Goyal. 2016. The Market Impacts of Pharmaceutical Product Patents in Developing Countries: Evidence from India. American Economic Review 106:01, 99-135. [Abstract] [View PDF article] [PDF with links]

2. Francesco Decarolis. 2015. Medicare Part D: Are Insurers Gaming the Low Income Subsidy Design?. American Economic Review 105:4, 1547-1580. [Abstract] [View PDF article] [PDF with links]

3. Keith M. Marzilli Ericson. 2014. Consumer Inertia and Firm Pricing in the Medicare Part D Prescription Drug Insurance Exchange. American Economic Journal: Economic Policy 6:1, 38-64. [Abstract] [View PDF article] [PDF with links]

4. Jason Abaluck, Jonathan Gruber. 2011. Heterogeneity in Choice Inconsistencies among the Elderly: Evidence from Prescription Drug Plan Choice. American Economic Review 101:3, 377-381. [Abstract] [View PDF article] [PDF with links] 